\title{
Derived invariants arising from the Albanese map
}

\author{
Federico Caucci and Giuseppe Pareschi
}

\begin{abstract}
Let $a_{X}: X \rightarrow \operatorname{Alb} X$ be the Albanese map of a smooth complex projective variety. Roughly speaking, in this note we prove that for all $i \geqslant 0$ and $\alpha \in \operatorname{Pic}^{0} X$, the cohomology ranks $h^{i}\left(\operatorname{Alb} X, a_{X *} \omega_{X} \otimes P_{\alpha}\right)$ are derived invariants. This proves conjectures of Popa and Lombardi-Popa - including the derived invariance of the Hodge numbers $h^{0, j}$-in the case of varieties of maximal Albanese dimension and a weaker version of them for arbitrary varieties. Finally, we provide an application to the derived invariance of certain irregular fibrations.
\end{abstract}

\section{Introduction}

This paper is about derived invariants of smooth complex projective varieties (henceforth called varieties) arising from the Albanese morphism

$$
a_{X}: X \rightarrow \operatorname{Alb} X
$$

For example, a fundamental result of Popa and Schnell shows that the dimension of the Albanese variety Alb $X$, that is, $h^{0}\left(\Omega_{X}^{1}\right)$, is a derived invariant [PS11].

Roughly speaking, in this note we prove that for all $i \geqslant 0$ and for all $\alpha \in \operatorname{Pic}^{0} X$, the cohomology ranks

$$
h^{i}\left(\operatorname{Alb} X, a_{X *} \omega_{X} \otimes P_{\alpha}\right)
$$

are derived invariants. This settles in the affirmative (a strengthened version of) a conjecture of Lombardi and Popa [LP15, Conjecture 11] - proved by Lombardi for $i=0$ and partially for $i=1$ (see [Lom14]) - in the case of varieties of maximal Albanese dimension and proves a weaker version of it for arbitrary varieties. (Recall that $X$ is said to have maximal Albanese dimension if $\operatorname{dim} a_{X}(X)=\operatorname{dim} X$.) For varieties of maximal Albanese dimension, this implies the derived invariance of the Hodge numbers $h^{0, j}$ for all $j \geqslant 0$ and of all canonical cohomological support loci, proving in this case another conjecture of Popa. In this direction, previous results in low dimension were obtained by Popa, Lombardi and Abuaf [Pop13, Lom14, LP15, Abu17].

Turning to precise statements, for a variety $X$, let us denote by $\mathbf{D}(X)$ its bounded derived category of coherent sheaves. Let $Y$ be another variety, and let

$$
\varphi: \mathbf{D}(X) \rightarrow \mathbf{D}(Y)
$$

be an exact equivalence. As shown by Rouquier [Rou11], see also [PS11], the equivalence $\varphi$

Received 11 November 2014, accepted in final form 26 November 2018.

2010 Mathematics Subject Classification 14F05 (primary), 14F17, 14E05, 14D06 (secondary).

Keywords: derived categories, cohomological support loci, Hodge numbers, fibrations.

This journal is (c) Foundation Compositio Mathematica 2019. This article is distributed with Open Access under the terms of the Creative Commons Attribution Non-Commercial License, which permits non-commercial reuse, distribution, and reproduction in any medium, provided that the original work is properly cited. For commercial re-use, please contact the Foundation Compositio Mathematica. 


\section{DERIVED INVARIANTS ARISING FROM THE Albanese MAP}

induces an isomorphism of algebraic groups

$$
\bar{\varphi}: \operatorname{Aut}^{0} X \times \operatorname{Pic}^{0} X \rightarrow \operatorname{Aut}^{0} Y \times \operatorname{Pic}^{0} Y .
$$

We choose normalized Poincaré line bundles so that to a closed point $\alpha \in \operatorname{Pic}^{0} X$ (respectively, $\beta \in \operatorname{Pic}^{0} Y$ ) corresponds the line bundle $P_{\alpha}$ on $X$ (respectively, $P_{\beta}$ on $Y$ ). Essential for our arguments is a result of Lombardi, from which it follows that if $h^{i}\left(\operatorname{Alb} X, a_{X *} \omega_{X} \otimes P_{\alpha}\right)>0$ for some $i \geqslant 0$, then $\bar{\varphi}\left(\operatorname{id}_{X}, P_{\alpha}\right)$ is of the form $\left(\operatorname{id}_{Y}, P_{\beta}\right)$ for a $\beta \in \operatorname{Pic}^{0} Y$. If this is the case, we will abusively write $\beta=\bar{\varphi}(\alpha)$.

TheOREM 1.1. Let $i \in \mathbb{N}$. In the above notation, $h^{i}\left(\operatorname{Alb} X, a_{X *} \omega_{X} \otimes P_{\alpha}\right)>0$ if and only if $h^{i}\left(\operatorname{Alb} Y, a_{Y *} \omega_{Y} \otimes P_{\bar{\varphi}(\alpha)}\right)>0$. If this is the case,

$$
h^{i}\left(\operatorname{Alb} X, a_{X *} \omega_{X} \otimes P_{\alpha}\right)=h^{i}\left(\operatorname{Alb} Y, a_{Y *} \omega_{Y} \otimes P_{\bar{\varphi}(\alpha)}\right) .
$$

It is expected that derived-equivalent varieties have the same Hodge numbers. As a consequence of the invariance of the cohomological ranks of the sheaves $a_{X *} \omega_{X}$, it follows that this holds true for the $h^{0, j}$ of varieties of maximal Albanese dimension.

Corollary 1.2. Let $X$ and $Y$ be smooth complex projective varieties with equivalent derived categories. Then, for all $i \in \mathbb{N}$,

$$
h^{i}\left(\operatorname{Alb} X, a_{X *} \omega_{X}\right)=h^{i}\left(\operatorname{Alb} Y, a_{Y *} \omega_{Y}\right) .
$$

In particular, if $X$ is of maximal Albanese dimension, then for all $j \geqslant 0$, we have

$$
h^{0, j}(X)=h^{0, j}(Y) .
$$

Notice that in the maximal Albanese dimension case, $R^{i} a_{X *} \omega_{X}=0$ for $i>0$ (GrauertRiemenschneider vanishing theorem) and therefore $h^{i}\left(X, \omega_{X}\right)=h^{i}\left(\operatorname{Alb} X, a_{X *} \omega_{X}\right)$. This proves the last part of the corollary.

Given a coherent sheaf $\mathcal{F}$ on a smooth projective variety $X$, its cohomological support loci are the following algebraic subvarieties of $\operatorname{Pic}^{0} X$ :

$$
V_{r}^{i}(X, \mathcal{F})=\left\{\alpha \in \operatorname{Pic}^{0} X \mid h^{i}\left(X, \mathcal{F} \otimes P_{\alpha}\right) \geqslant r\right\} .
$$

For $r=1$, we simply set $V_{r}^{i}(X, \mathcal{F})=V^{i}(X, \mathcal{F})$. Again, by the Grauert-Riemenschneider vanishing theorem and the projection formula, it follows that $V_{r}^{i}\left(X, \omega_{X}\right)=V_{r}^{i}\left(\operatorname{Alb} X, a_{X *} \omega_{X}\right)$ in the maximal Albanese dimension case.

It has been conjectured by Popa [Pop13] that all loci $V^{i}\left(X, \omega_{X}\right)$ are derived invariants of smooth complex projective varieties. This conjecture has been verified by Lombardi and Popa, only for the components containing the origin of $\mathrm{Pic}^{0} X$, unconditionally on the Albanese dimension for $i=0,1, \operatorname{dim} X-1, \operatorname{dim} X$ (see [Lom14, LP15]) and in dimension 3 (see [Lom14]), and for varieties of maximal Albanese dimension in dimension 4 (see [LP15]). The following corollary fully proves Popa's conjecture for varieties of maximal Albanese dimension and, in general, the analogous statement for the loci $V_{r}^{i}\left(\operatorname{Alb} X, a_{X *} \omega_{X}\right)$.

Corollary 1.3. Let $X$ and $Y$ be varieties with equivalent derived categories. For all $i, r \in \mathbb{N}$, the Rouquier isomorphism induces an isomorphism between $V_{r}^{i}\left(\operatorname{Alb} X, a_{X *} \omega_{X}\right)$ and $V_{r}^{i}\left(\operatorname{Alb} Y, a_{Y *} \omega_{Y}\right)$.

In particular, if $X$ is of maximal Albanese dimension, then for every choice of $i, r \in \mathbb{N}$, the cohomological support loci $V_{r}^{i}\left(X, \omega_{X}\right)$ and $V_{r}^{i}\left(Y, \omega_{Y}\right)$ are isomorphic.

The method of proof of Theorem 1.1 makes use of many essential results concerning the geometry of irregular varieties based on generic vanishing theory: generic vanishing theorems, the 


\section{F. Caucci and G. Pareschi}

relation between the loci $V^{0}\left(X, \omega_{X}^{m}\right)$ and the Iitaka fibration, the Chen-Jiang decomposition, linearity theorems and their relation - via the Bernstein-Gel'fand-Gel'fand correspondencewith the Castelnuovo-Mumford regularity of suitable cohomology modules. This material is briefly reviewed in Sections 2 and 3. The starting point of the argument are the results and constructions of Lombardi [Lom14], who proves, in particular, the case $i=0$ of Theorem 1.1. Roughly, our method derives Theorem 1.1 from the case $i=0$ by means of the derived invariance of the Hochschild multiplicative structure, combined with the result of Lazarsfeld, Popa and Schnell on the cohomology modules $H^{*}\left(\operatorname{Alb} X, a_{X *} \omega_{X} \otimes P_{\alpha}\right)$ over the exterior algebra $\Lambda^{*} H^{1}\left(\operatorname{Alb} X, \mathcal{O}_{\operatorname{Alb} X}\right)$.

Next, we turn to some applications of Theorem 1.1 and especially of Corollary 1.3. It is known by the seminal work of Green and Lazarsfeld [GL91] that the positive-dimensional components of the loci $V_{r}^{i}\left(X, \omega_{X}\right)$ are related to the presence of irregular fibrations, that is, morphisms with connected fibres onto lower-dimensional normal projective varieties, which we call the bases of the fibrations, whose smooth models have maximal Albanese dimension. Therefore, as sought by Popa [Pop13] and in the spirit of previous work of Lombardi and Popa [Lom14, LP15] (and especially [Lom18]), the part of Corollary 1.3 concerning varieties of maximal Albanese dimension implies the derived invariance of the presence or absence of certain irregular fibrations and, moreover, the invariance of the set itself of such fibrations. This imposes striking restrictions to the geometry and topology of the Fourier-Mukai partners. An example of this is Theorem 1.4 concerning irregular fibrations of minimal base dimension on varieties of maximal Albanese dimension. We remark that it is likely that a more thorough analysis of the information provided by Theorem 1.1 can lead to more complete results.

Turning to details, let us recall some notions appearing in the statement of Theorem 1.4. In the first place, we recall that $\chi\left(\omega_{Z}\right) \geqslant 0$ for a variety $Z$ of maximal Albanese dimension (see Section 2). An irregular fibration

$$
g: X \rightarrow S
$$

is said to be $\chi$-positive if $\chi\left(\omega_{S^{\prime}}\right)>0$ for a smooth model $S^{\prime}$ of $S$ (hence for all of them). This implies, in particular, that $S^{\prime}$ is of general type. One might see $\chi$-positive fibrations as the higherdimensional analogue of fibrations onto curves of genus at least 2 , which were classically studied by Castelnuovo and de Franchis [Cas05, dFr05]. Unconditionally on the Albanese dimension, Lombardi proved the invariance of the equivalence classes of the set of fibrations over curves of genus at least 2 (see [Lom18]). For varieties of maximal Albanese dimension, we note that as a consequence of Orlov's theorem on the derived invariance of the canonical ring, the equivalence classes of all $\chi$-positive irregular fibrations are derived invariant (Proposition 4.7 below).

On the other hand, even in the case of varieties of maximal Albanese dimension, it is unclear what happens for non- $\chi$-positive fibrations, especially when the base is birational to an abelian variety. Theorem 1.4 gives a positive result about the derived invariance of the equivalence classes of a certain type of irregular fibrations which are not necessarily $\chi$-positive and include certain fibrations onto abelian varieties. In order to state the result, let us denote by $\operatorname{Pic}^{0}(g)$ the kernel of the restriction of $\operatorname{Pic}^{0} X$ to a general fibre of $g$. It is well known that $\operatorname{Pic}^{0}(g)$ is an extension of $g^{*} \mathrm{Pic}^{0} S$ by a finite subgroup of $\mathrm{Pic}^{0} X / g^{*} \mathrm{Pic}^{0} S$; hence, it may be disconnected. (For fibrations $g$ onto curves, the subvariety $\operatorname{Pic}^{0}(g)$ is completely described in the work of Beauville [Bea92].) We call cohomologically detectable all irregular fibrations $g: X \rightarrow S$ except those such that $S$ is birational to an abelian variety $B$ with $\operatorname{Pic}^{0}(g)=g^{*} \operatorname{Pic}^{0} B$, and we denote by $b(X)$ the minimal base dimension (namely $\operatorname{dim} S$ ) of such fibrations (if there are no cohomologically detectable fibrations, we declare that $b(X)=0$ ). The explanation for such terminology is in Remark 4.6. 


\section{DERIVED INVARIANTS ARISING FROM THE Albanese MAP}

Here, it suffices to say that by the Green-Lazarsfeld linearity theorem, "most" irregular fibrations are induced by positive-dimensional components of the various loci $V^{i}\left(X, \omega_{X}\right)$. For irregular fibrations $g$ whose base is birational to an abelian variety and for which $\operatorname{Pic}^{0}(g)$ is connected, either this does not happen, or it happens in a non-standard way.

Theorem 1.4. Let $X$ and $Y$ be $d$-dimensional derived-equivalent varieties of maximal Albanese dimension. Then $b(X)=b(Y)=: b$. Moreover there is a base-preserving bijection of the sets of the equivalence classes of cohomologically detectable irregular fibrations of $X$ and $Y$ of base dimension equal to $b$. Such a bijection takes $\chi$-positive fibrations to $\chi$-positive fibrations.

Theorem 1.4 is proved by considering a special class of components of the loci $V^{i}\left(X, \omega_{X}\right)$, studied by the second author in [Par17], whose relation with the corresponding fibration (via the theorem of Green and Lazarsfeld) is somewhat standard. We first show a fact of independent interest (Lemma 4.5), namely that these "standard" components are translates of abelian subvarieties for which there is a natural bijective correspondence with the set of equivalence classes of another type of fibrations, called weakly- $\chi$-positive, containing the $\chi$-positive ones. However, in general there is no easy way to distinguish the standard components from the non-standard ones. In the second part of the proof of Theorem 1.4, we show that this can be done in the locus $V^{d-b(X)}\left(X, \omega_{X}\right)$, and in this case, the weakly- $\chi$-positive fibrations coincide with the cohomologically detectable ones. In this way, Theorem 1.4 follows from Corollary 1.3.

Finally, we remark that Theorem 1.1 also provides some information about the derived invariance of fibrations of varieties of arbitrary Albanese dimension. In fact, a well-known argument using Kollár decomposition shows that positive-dimensional irreducible components of the loci $V_{r}^{i}\left(\operatorname{Alb} X, a_{X *} \omega_{X}\right)$ form a subset of the set of the irreducible components of the loci $V_{r^{\prime}}^{i}\left(X, \omega_{X}\right)$ for some $r^{\prime} \geqslant r$. Hence, via the Green-Lazarsfeld theorem, they correspond to some irregular fibrations. However, at present it is not clear to us how to describe them.

We will work over $\mathbb{C}$. All varieties appearing in this paper are assumed to be projective. A variety without further specification is a smooth complex projective variety. Normal variety means normal projective variety. An Albanese morphism means a universal morphism from a fixed variety $X$ to abelian varieties. We will call such a morphism the Albanese morphism or also the Albanese map of $X$, and we will denote it by $a_{X}: X \rightarrow \operatorname{Alb} X$.

\section{Preliminary material on generic vanishing, Chen-Jiang decomposition and 0-regularity of the canonical module}

In this section, we recall material used further on; we refer to the appropriate sections of papers such as [Par12, HPS18, PS13, PPS17, Par17] for more thorough surveys. For a morphism of abelian varieties $\pi: A \rightarrow B$, we will denote the dual morphism by

$$
\widehat{\pi}: \operatorname{Pic}^{0} B \rightarrow \operatorname{Pic}^{0} A \text {. }
$$

Generic vanishing. Let $A$ be an abelian variety. A coherent sheaf $\mathcal{G}$ is said to be a generic vanishing sheaf, or $G V$-sheaf for short, if

$$
\operatorname{codim}_{\mathrm{Pic}^{0} A} V^{i}(A, \mathcal{G}) \geqslant i \text { for all } i \geqslant 0 .
$$

The sheaf $\mathcal{G}$ is said to be $M$-regular if

$$
\operatorname{codim}_{\mathrm{Pic}^{0} A} V^{i}(A, \mathcal{G})>i \text { for all } i>0 .
$$




\section{F. CAUCCI AND G. PAREschi}

Remark 2.1. If $\mathcal{G}$ is $\mathrm{GV}$, then $\chi(\mathcal{G}) \geqslant 0$ and $\chi(\mathcal{G})>0$ if and only if $V^{0}(A, \mathcal{G})=\operatorname{Pic}^{0} A$.

We have the following well-known non-vanishing results (see, for example, [Hac04, Corollary 3.2] for part (a) and [Par12, Lemma 1.12] for parts (b) and (c))

Proposition 2.2. Let $\mathcal{G}$ be a non-zero coherent sheaf on an abelian variety $A$.

(a) If $\mathcal{G}$ is $G V$, then $V^{i+1}(A, \mathcal{G}) \subseteq V^{i}(A, \mathcal{G})$ for all $i \geqslant 0$.

(b) If $\mathcal{G}$ is $G V$, then $V^{0}(A, \mathcal{G}) \neq \emptyset$.

(c) If $\mathcal{G}$ is M-regular, then $V^{0}(A, \mathcal{G})=\operatorname{Pic}^{0} A$.

Chen-Jiang decomposition. This concept was introduced by Chen and Jiang [CJ18, Theorem 1.1]. The following theorem was proved in [PPS17]. In this paper, we will use only the case $j=0$.

Theorem 2.3 (Chen-Jiang decomposition). Let $a: X \rightarrow A$ be a morphism from a variety to an abelian variety, and let $j \geqslant 0$. Then the sheaf $R^{j} a_{*} \omega_{X}$ decomposes canonically as

$$
R^{j} a_{*} \omega_{X}=\bigoplus_{i} \pi_{i}^{*} \mathcal{F}_{i} \otimes P_{\alpha_{i}}
$$

where the $\pi_{i}: A \rightarrow B_{i}$ are quotients of abelian varieties with connected fibres, the $\mathcal{F}_{i}$ are $M$ regular sheaves on $B_{i}$ and the $\alpha_{i}$ are torsion points of $\mathrm{Pic}^{0} A$.

Note that in the above decomposition, we can arrange that $\widehat{\pi}_{i}\left(\operatorname{Pic}^{0} B_{i}\right)-\alpha_{i} \neq \widehat{\pi}_{k}\left(\operatorname{Pic}^{0} B_{k}\right)-$ $\alpha_{k}$ for $i \neq k$. With this normalization, the decomposition is canonical up to permutation of the summands.

Remark 2.4. Theorem 2.3 has the following consequences:

(1) For all $j \geqslant 0$, the sheaf $R^{j} a_{*} \omega_{X}$ is a $G V$-sheaf on $A$ (Hacon [Hac04]). This is because, by the projection formula, the pullback of a GV-sheaf via a morphism of abelian varieties is still GV.

(2) $V^{0}\left(A, R^{j} a_{*} \omega_{X}\right)=\bigcup_{i}\left(\widehat{\pi}_{i}\left(\operatorname{Pic}^{0} B_{i}\right)-\alpha_{i}\right)$. This equality again follows from the projection formula:

$H^{0}\left(A, \pi_{i}^{*} \mathcal{F}_{i} \otimes P_{\alpha_{i}} \otimes P_{\alpha}\right)= \begin{cases}H^{0}\left(B_{i}, \mathcal{F}_{i} \otimes P_{\beta}\right) & \text { if } \alpha=\widehat{\pi}_{i}(\beta)-\alpha_{i} \text { with } \beta \in \widehat{\pi}_{i}\left(\operatorname{Pic}^{0} B_{i}\right), \\ 0 & \text { otherwise. }\end{cases}$

This, together with Proposition 2.2(c), shows that the locus $V^{0}\left(A, R^{j} a_{*} \omega_{X}\right)$ is the union of translates of the abelian subvarieties $\widehat{\pi}_{i}\left(\mathrm{Pic}^{0} B_{i}\right)$ of $\mathrm{Pic}^{0} A$ by points of finite order. ${ }^{1}$

(3) Keeping the notation of Theorem 2.3, let $c(i)=\operatorname{dim} A-\operatorname{dim} B_{i}$. Again from the projection formula, combined with Proposition 2.2(c), it follows that the support of $V^{c(i)}\left(A, \pi_{i}^{*} \mathcal{F}_{i} \otimes P_{\alpha_{i}}\right)$ is equal to the support of $V^{0}\left(A, \pi_{i}^{*} \mathcal{F}_{i} \otimes P_{\alpha_{i}}\right)$, namely $\widehat{\pi}_{i}\left(\operatorname{Pic}^{0} B_{i}\right)-\alpha_{i}$. This implies a result originally due to Ein-Lazarsfeld [EL97]: the irreducible components of the locus $V^{0}\left(A, R^{j} a_{*} \omega_{X}\right)$ of codimension $c>0$ are also components of the locus $V^{c}\left(A, R^{j} a_{*} \omega_{X}\right)$.

Remark 2.5. Theorem 2.3 and its consequences hold more generally for the sheaves $R^{j} a_{*}\left(\omega_{X} \otimes\right.$ $P_{\alpha}$ ), where $\alpha$ is a torsion point of $\operatorname{Pic}^{0} X$. This is because $\omega_{X} \otimes P_{\alpha}$ is a direct summand of $f_{*} \omega_{\widetilde{X}}$ for a suitable étale cover $f: \widetilde{X} \rightarrow X$.

${ }^{1}$ By a theorem of Green-Lazarsfeld and Simpson, this is actually true, and of fundamental importance, for all loci $V_{r}^{i}\left(A, R^{j} a_{*} \omega_{X}\right)$ for all $i, j$ and $r$; see Section 4 . 


\section{DERIVED INVARIANTS ARISING FROM THE Albanese MAP}

Strong linearity and Castelnuovo-Mumford regularity. The relation between the theory of generic vanishing and the Bernstein-Gel'fand-Gel'fand correspondence was pointed out in the paper [LP10] and further developed in [LPS11] and [PS13].

For a sheaf $\mathcal{G}$ on an abelian variety $A$, let us consider its cohomology module

$$
H^{*}(A, \mathcal{G})=\bigoplus_{i} H^{i}(A, \mathcal{G})
$$

which is a graded module over the exterior algebra $\Lambda^{*} H^{1}\left(A, \mathcal{O}_{A}\right)$. For such a graded module, there is the notion of Castelnuovo-Mumford regularity. In particular, $\operatorname{reg}\left(H^{*}(A, \mathcal{G})\right)=0$ if and only if it is generated in degree 0 and it has a linear graded free resolution. We will use the case $j=0$ of the following theorem of Lazarsfeld, Popa and Schnell [LPS11, Theorem 2.1].

Theorem 2.6 (Lazarsfeld-Popa-Schnell). Let $a: X \rightarrow A$ be a morphism from a variety $X$ to an abelian variety $A$. Let $\alpha \in \operatorname{Pic}^{0} X$ be a torsion point, and let $\beta \in \operatorname{Pic}^{0} A$. Then, for all $j \geqslant 0$,

$$
\operatorname{reg}\left(H^{*}\left(A, R^{j} a_{*}\left(\omega_{X} \otimes P_{\alpha}\right) \otimes P_{\beta}\right)\right)=0 .
$$

Note that this theorem is stated in [LPS11] in a more restrictive setting, namely only for $R^{j} a_{X *} \omega_{X}$, where $a_{X}$ denotes the Albanese morphism of $X$. However, the proof of the result goes through without any changes. The point here is that the Green-Lazarsfeld theorem about computing the higher direct images of the Poincaré bundle by means of the derivative complex [GL91, §3] holds in the neighbourhood of every point in $\operatorname{Pic}^{0} A$, so that the machinery in [LP10] and [LPS11] applies.

\section{Proof of Theorem 1.1}

Preliminaries: Two results of Lombardi. Again, for sake of brevity, we will state only those results strictly needed for our arguments, referring to the paper [Lom14] for the complete story. Let $\varphi: \mathbf{D}(X) \rightarrow \mathbf{D}(Y)$ be an exact equivalence and

$$
\bar{\varphi}: \operatorname{Aut}^{0} X \times \operatorname{Pic}^{0} X \rightarrow \operatorname{Aut}^{0} Y \times \operatorname{Pic}^{0} Y
$$

its Rouquier isomorphism. The following result [Lom14, Proposition 3.1] will be fundamental for our arguments.

Theorem 3.1. Let $m$ be an integer, and assume $h^{0}\left(X, \omega_{X}^{m} \otimes P_{\alpha}\right)>0$. Then $\bar{\varphi}\left(\mathrm{id}_{X}, P_{\alpha}\right)$ is of the form $\left(\operatorname{id}_{Y}, P_{\beta}\right)$ for $\beta \in \operatorname{Pic}^{0} Y$. If this is the case, we will abusively write

$$
\beta=\bar{\varphi}(\alpha) .
$$

Let us denote by $\delta: X \rightarrow X \times X$ the diagonal morphism. Again, following Lombardi [Lom14], for fixed $m \in \mathbb{Z}$ and $\alpha \in \mathrm{Pic}^{0} X$, we consider the twisted (generalized) Hochschild homology ${ }^{2}$

$$
H H_{*}^{m}(X, \alpha)=\bigoplus_{k} \operatorname{Ext}_{\mathcal{O}_{X \times X}}^{k}\left(\delta_{*} \mathcal{O}_{X}, \delta_{*}\left(\omega_{X}^{m} \otimes P_{\alpha}\right)\right)
$$

It is a graded module over the Hochschild cohomology algebra

$$
H H^{*}(X)=\bigoplus_{k} \operatorname{Ext}_{\mathcal{O}_{X \times X}}^{k}\left(\delta_{*} \mathcal{O}_{X}, \delta_{*} \mathcal{O}_{X}\right)
$$

\footnotetext{
${ }^{2}$ As already mentioned, Lombardi's setting is more general. Here, we are stating only what is necessary for our purposes.
} 


\section{F. CAucci and G. Pareschi}

The following is a classical result of Orlov and Căldăraru [Orl03, Căl03], generalized by Lombardi to the twisted case [Lom14, Theorem 1.1].

Theorem 3.2. In the above notation, let $m \in \mathbb{Z}$ and $\alpha \in \operatorname{Pic}^{0} X$ be such that $h^{0}\left(X, \omega_{X}^{m} \otimes P_{\alpha}\right)>0$. Then the derived equivalence $\varphi$ induces a canonical graded-algebra isomorphism

$$
\Phi^{*}: H H^{*}(X) \rightarrow H H^{*}(Y)
$$

and, using notation (3.1), a compatible graded-module isomorphism

$$
\Phi_{\alpha, *}^{m}: H H_{*}^{m}(X, \alpha) \rightarrow H H_{*}^{m}(Y, \bar{\varphi}(\alpha)) .
$$

In particular, in degree $0, H H_{0}^{m}(X, \alpha)=H^{0}\left(X, \omega_{X}^{m} \otimes P_{\alpha}\right)$; hence, we have the isomorphism

$$
\Phi_{\alpha, 0}^{m}: H^{0}\left(X, \omega_{X}^{m} \otimes P_{\alpha}\right) \stackrel{\sim}{\rightarrow} H^{0}\left(Y, \omega_{Y}^{m} \otimes P_{\bar{\varphi}(\alpha)}\right) .
$$

Going back to the Rouquier isomorphism, it follows that for all $m \in \mathbb{Z}$ and $r \geqslant 1$,

$$
\bar{\varphi}\left(\left\{\operatorname{id}_{X}\right\} \times V_{r}^{0}\left(X, \omega_{X}^{m}\right)\right)=\left\{\operatorname{id}_{Y}\right\} \times V_{r}^{0}\left(Y, \omega_{Y}^{m}\right) .
$$

For $m=1$, we will suppress, as is customary, the index 1 in (3.2) and in (3.3).

Preliminaries: The Iitaka fibration of irregular varieties. Assume that the Kodaira dimension of $X$ is non-negative. Then the loci $V^{0}\left(X, \omega_{X}^{m}\right)$ are tightly connected with the Iitaka fibration of $X$.

After a birational modification of $X$, we can assume that the Iitaka fibration of $X$ is a morphism $X \rightarrow Z_{X}$ with $Z_{X}$ smooth. There is the commutative diagram

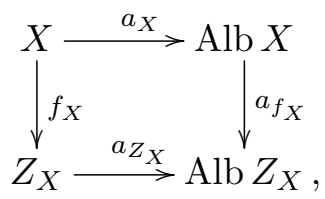

where $a_{f_{X}}$ is a surjective morphism of abelian varieties with connected fibres [HPS18, Lemma 1.11(a)]. We will use the following results of Chen-Hacon and Hacon-Popa-Schnell.

TheOREM 3.3. (a) ([HPS18, Theorem 11.2(b)]) For $m \geqslant 2$, the irreducible components of the locus $V^{0}\left(X, \omega_{X}^{m}\right)$ are translates of $\widehat{a_{f_{X}}}\left(\operatorname{Pic}^{0} Z_{X}\right)$ by torsion points of $\operatorname{Pic}^{0} X$.

(b) ([CH01b, Lemma 2.2], see also [HPS18, (2) after Lemma 11.1]) The irreducible components of the locus $V^{0}\left(X, \omega_{X}\right)$ are translates of abelian subvarieties of the abelian subvariety $\widehat{a_{f_{X}}}\left(\mathrm{Pic}^{0} Z_{X}\right)$ by torsion points of $\operatorname{Pic}^{0} X$.

Proof of Theorem 1.1. Let $\alpha \in \operatorname{Pic}^{0} X$ and $i \geqslant 0$ be such that

$$
h^{i}\left(\operatorname{Alb} X, a_{X *} \omega_{X} \otimes P_{\alpha}\right)>0 .
$$

Step 1: The Kodaira dimensions of $X$ and $Y$ are non-negative.

Proof. Indeed, by (3.6), we have $V^{i}\left(\operatorname{Alb} X, a_{X *} \omega_{X}\right) \neq \emptyset$. Therefore, $V^{0}\left(\operatorname{Alb} X, a_{X *} \omega_{X}\right) \neq \emptyset$ by Proposition 2.2(a). By Remark 2.4(2), this yields that $V^{0}\left(\operatorname{Alb} X, a_{X *} \omega_{X}\right)=V^{0}\left(X, \omega_{X}\right)$ contains some points $\alpha$ of $\operatorname{Pic}^{0} X$ of finite order, say $k$. This implies that we have $h^{0}\left(X,\left(\omega_{X} \otimes P_{\alpha}\right)^{k}\right)=$ $h^{0}\left(X, \omega_{X}^{k}\right)>0$. Therefore, $\kappa(X) \geqslant 0$. Since the Kodaira dimension is a derived invariant, the same holds for $Y$. 


\section{DeRIVED INVARIANTS ARISING FROM the Albanese MAP}

We have natural embeddings

$$
H^{1}\left(Z_{X}, \mathcal{O}_{Z_{X}}\right) \subset H^{1}\left(X, \mathcal{O}_{X}\right) \subset H H^{1}(X)=\operatorname{Ext}_{\mathcal{O}_{X \times X}}^{1}\left(\delta_{*} \mathcal{O}_{X}, \delta_{*} \mathcal{O}_{X}\right)
$$

The same holds for $Y$.

Step 2: The second step is the following.

Lemma 3.4. We have the identity $\Phi^{1} H^{1}\left(Z_{X}, \mathcal{O}_{Z_{X}}\right)=H^{1}\left(Z_{Y}, \mathcal{O}_{Z_{Y}}\right)$.

Proof. This follows at once from the above results. Indeed, combining Theorem 3.3(a) and (3.5), we get that

$$
\bar{\varphi}\left(\widehat{a_{f_{X}}}\left(\operatorname{Pic}^{0} Z_{X}\right)\right)=\widehat{a_{f_{Y}}}\left(\operatorname{Pic}^{0} Z_{Y}\right) .
$$

On the other hand, it is well known that the isomorphism $\Phi^{1}$, that is, ${ }^{3}$

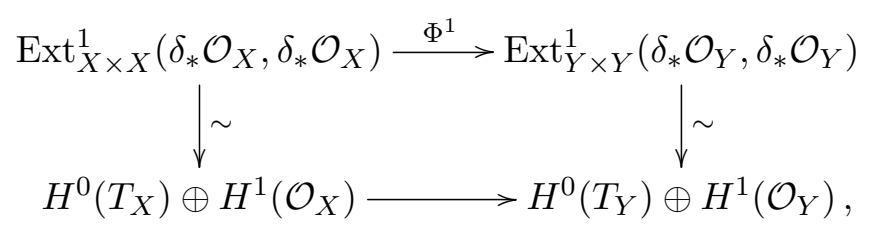

is the first-order version of the Rouquier isomorphism (see, for example, [Huy06, discussion after Proposition 9.45, p. 218]). Therefore, Step 2 follows from (3.7).

Next, we note that, by Theorem 3.3(b), we can gather those irreducible components of $V^{0}\left(X, \omega_{X}\right)=V^{0}\left(\operatorname{Alb} X, a_{X *} \omega_{X}\right)$ which are contained in the same translate of $\widehat{a_{f_{X}}}\left(\operatorname{Pic}^{0} Z_{X}\right)$. Hence, using Remark 2.4(2), we can gather the corresponding sheaves appearing in the ChenJiang decomposition of $a_{X *} \omega_{X}$, yielding another canonical decomposition

$$
a_{X *} \omega_{X}=\bigoplus_{j=1}^{r_{X}}\left(a_{f_{X}}^{*} \mathcal{H}_{X, j}\right) \otimes P_{X, \delta_{j}}
$$

defined by the following properties:

The $\mathcal{H}_{X, j}$ are GV-sheaves on $\operatorname{Alb} Z_{X}$ (in fact, the direct sum of some pullbacks of $M$ regular sheaves from quotient abelian varieties appearing in the Chen-Jiang decomposition of $\left.a_{X *} \omega_{X}\right)$. The $\delta_{j}$ are torsion points of $\operatorname{Pic}^{0} X$, and $r_{X}$ is the number of translates in $\mathrm{Pic}^{0} X$ of the abelian subvariety $\widehat{a_{f_{X}}}\left(\operatorname{Pic}^{0} Z_{X}\right)$ containing at least one component of the locus $V^{0}\left(\operatorname{Alb} X, a_{X *} \omega_{X}\right)$.

The same sort of decomposition holds for $a_{Y *} \omega_{Y}$ :

$$
a_{Y *} \omega_{Y}=\bigoplus_{k=1}^{r_{Y}}\left(a_{f_{Y}}^{*} \mathcal{H}_{Y, k}\right) \otimes P_{Y, \gamma_{k}} .
$$

We claim that $r_{X}=r_{Y}:=r$ and, up to reordering, for all $j=1, \ldots, r$,

$$
\bar{\varphi}\left(V^{0}\left(X, \omega_{X}\right) \cap\left(\widehat{a_{f_{X}}}\left(\operatorname{Pic}^{0} Z_{X}\right)-\delta_{j}\right)\right)=V^{0}\left(Y, \omega_{Y}\right) \cap\left(\widehat{a_{f_{Y}}}\left(\operatorname{Pic}^{0} Z_{Y}\right)-\gamma_{j}\right) .
$$

In fact, each component of $V^{0}\left(X, \omega_{X}\right)$ (which is a translate of an abelian subvariety $\widehat{a_{f_{X}}}\left(\operatorname{Pic}^{0} Z_{X}\right)$ ) is contained in a unique translate of $\widehat{a_{f_{X}}}\left(\mathrm{Pic}^{0} Z_{X}\right)$. The same happens on $Y$. From $(3.7)$ and

\footnotetext{
${ }^{3}$ The spectral sequence abutting to $\operatorname{Ext}_{X \times X}^{i}\left(\delta_{*} \mathcal{O}_{X}, \delta_{*} \mathcal{O}_{X}\right)$ degenerates; see [Swa96, Corollary 2.6].
} 


\section{F. CAucci and G. Pareschi}

Lombardi's theorem (3.5), it follows that the algebraic group isomorphism $\bar{\varphi}$ sends such a translate of $\widehat{a_{f_{X}}}\left(\operatorname{Pic}^{0} Z_{X}\right)$ to the corresponding translate of $\widehat{a_{f_{Y}}}\left(\operatorname{Pic}^{0} Z_{Y}\right)$ in $\operatorname{Pic}^{0} Y$. This proves what we claimed.

Since two different translates have empty intersection, we have that

(*) for $i \geqslant 0$ and for a fixed $\alpha \in \mathrm{Pic}^{0} X$, in the decomposition

$$
H^{i}\left(a_{X *} \omega_{X} \otimes P_{\alpha}\right)=\bigoplus_{j=1}^{r_{X}} H^{i}\left(\left(a_{f_{X}}^{*} \mathcal{H}_{X, j}\right) \otimes P_{\delta_{j}+\alpha}\right),
$$

at most one summand is non-zero.

For $i=0$, this holds by the definition of the above decomposition, and for $i>0$, it follows as above from Proposition 2.2(a). Moreover, from the projection formula and the fact that the quotient $\operatorname{Alb} X \rightarrow \operatorname{Alb} Z_{X}$ has connected fibres, it follows that

$$
\begin{aligned}
H^{0}( & \left.\operatorname{Alb} X,\left(a_{f_{X}}^{*} \mathcal{H}_{X, j}\right) \otimes P_{\delta_{j}+\alpha}\right) \\
& = \begin{cases}H^{0}\left(\operatorname{Alb} Z_{X}, \mathcal{H}_{X, j} \otimes P_{\eta}\right) & \text { if } \delta_{j}+\alpha=\widehat{a_{f_{X}}}(\eta) \text { with } \eta \in \operatorname{Pic}^{0} Z_{X}, \\
0, & \text { otherwise. }\end{cases}
\end{aligned}
$$

The same holds for $Y$. This, combined with (3.4) proves the following.

Step 3: Keeping the above notation, let $\alpha \in V^{0}\left(\operatorname{Alb} X, a_{X *} \omega_{X}\right)$ and $\eta \in \operatorname{Pic}^{0} Z_{X}$ be such that $\widehat{a_{f_{X}}}(\eta)=\alpha+\delta_{j}$. Then

$$
\Phi_{\alpha+\delta_{j}}^{0} H^{0}\left(\operatorname{Alb} Z_{X}, \mathcal{H}_{X, j} \otimes P_{\eta}\right)=H^{0}\left(\operatorname{Alb} Z_{Y}, \mathcal{H}_{Y, j} \otimes P_{\bar{\varphi}(\eta)}\right),
$$

where, via a slight abuse of language, we denote by $\bar{\varphi}(\eta) \in \operatorname{Pic}^{0} Z_{Y}$ the element $\nu \in \operatorname{Pic}^{0} Z_{Y}$ such that, by (3.7), we have $\widehat{a_{f_{Y}}}(\nu)=\bar{\varphi}\left(\widehat{a_{f_{X}}}(\eta)\right)$.

Next, we recall that for all $\alpha \in \operatorname{Pic}^{0} X$, the local-to-global spectral sequence computing each graded component $H_{i}(X, \alpha)$ degenerates [Swa96, Corollary 2.6]. It follows that the canonical map from $H^{i}\left(X, \omega_{X} \otimes P_{\alpha}\right)$ to $H H_{i}(X, \alpha)$ is an embedding. Moreover, for $\alpha \in V^{0}\left(\operatorname{Alb} X, a_{X *} \omega_{X}\right)$ and $\eta \in \operatorname{Pic}^{0} Z_{X}$ such that $\widehat{a_{f_{X}}}(\eta)=\alpha+\delta_{j}$, we have the following chain of canonical embeddings of vector spaces:

$$
H^{i}\left(\operatorname{Alb} Z_{X}, \mathcal{H}_{X, j} \otimes P_{\eta}\right) \hookrightarrow H^{i}\left(\operatorname{Alb} X, a_{X *} \omega_{X} \otimes P_{\alpha}\right) \hookrightarrow H^{i}\left(X, \omega_{X} \otimes P_{\alpha}\right) \hookrightarrow H H_{i}(X, \alpha)
$$

(and the same things holds for $Y) .{ }^{4}$ The first inclusion follows from (3.8) via the projection formula, and the second one follows from Kollár's theorem on the degeneration of the Leray spectral sequence of the canonical bundle [Kol86b], once again combined with the projection formula.

Step 4: Let $\alpha \in V^{0}\left(\operatorname{Alb} X, a_{X *} \omega_{X}\right)$ and $\eta \in \operatorname{Pic}^{0} Z_{X}$ be such that $\widehat{a_{f_{X}}}(\eta)=\alpha+\delta_{j}$. Then, for all $i \geqslant 0$,

$$
\Phi_{\alpha+\delta_{j}}^{i} H^{i}\left(\operatorname{Alb} Z_{X}, \mathcal{H}_{X, j} \otimes P_{\eta}\right)=H^{i}\left(\operatorname{Alb} Z_{Y}, \mathcal{H}_{Y, j} \otimes P_{\bar{\varphi}(\eta)}\right)
$$

Proof. It is here that we use the multiplicative structure and the Bernstein-Gel'fand-Gel'fand

\footnotetext{
${ }^{4}$ In the second space, $P_{\alpha}$ denotes a line bundle on Alb $X$, while in the third space, $P_{\alpha}$ denotes a line bundle on $X$, that is, strictly speaking, the pullback, via the Albanese map, of the previous $P_{\alpha}$.
} 


\section{DeRIVED INVARIANTS ARISING FROM the Albanese MAP}

correspondence. We have the following morphisms of graded algebras:

$$
\Lambda^{*} H^{1}\left(Z_{X}, \mathcal{O}_{Z_{X}}\right) \hookrightarrow \Lambda^{*} H^{1}\left(X, \mathcal{O}_{X}\right) \rightarrow H^{*}\left(X, \mathcal{O}_{X}\right) \hookrightarrow H H^{*}(X) .
$$

Therefore, the Hochschild cohomology $H H_{*}(X, \alpha)$ is a graded module also on all the graded algebras appearing above. The similar result holds for $H H_{*}(Y, \beta)$. From Step 2, it follows that

(**) the graded-module isomorphism $\Phi_{\alpha}: H H_{*}(X, \alpha) \stackrel{\sim}{\rightarrow} H H_{*}(Y, \bar{\varphi}(\alpha))$ of Theorem 3.2 is compatible with the isomorphism $\wedge^{*}\left(\Phi^{1}\right): \Lambda^{*} H^{1}\left(\mathcal{O}_{Z_{X}}\right) \stackrel{\sim}{\rightarrow} \Lambda^{*} H^{1}\left(\mathcal{O}_{Z_{Y}}\right)$.

The inclusions (3.10) fit into inclusions of graded modules over the exterior algebra $\Lambda^{*} H^{1}\left(\mathcal{O}_{Z_{X}}\right)$ :

$$
H^{*}\left(\operatorname{Alb} Z_{X}, \mathcal{H}_{X, j} \otimes P_{\eta}\right) \hookrightarrow H^{*}\left(\operatorname{Alb} X, a_{X *} \omega_{X} \otimes P_{\alpha}\right) \hookrightarrow H^{*}\left(X, \omega_{X} \otimes P_{\alpha}\right) \hookrightarrow H H_{*}(X, \alpha) .
$$

For $\alpha \in V^{0}\left(\operatorname{Alb} X, a_{X *} \omega_{X}\right)$ and $\eta \in \operatorname{Pic}^{0} Z_{X}$ such that $\widehat{a_{f_{X}}}(\eta)=\alpha+\delta_{j}$, let us denote by

$$
\widetilde{H}^{*}\left(\operatorname{Alb} Z_{X}, \mathcal{H}_{X, j} \otimes P_{\eta}\right)
$$

the graded $\Lambda^{*} H^{1}\left(\mathcal{O}_{Z_{X}}\right)$-submodule of $H H_{*}(X, \alpha)$ generated by $H^{0}\left(\operatorname{Alb} Z_{X}, \mathcal{H}_{X, j} \otimes P_{\eta}\right)$. Clearly,

$$
\widetilde{H}^{*}\left(\operatorname{Alb} Z_{X}, \mathcal{H}_{X, j} \otimes P_{\eta}\right) \subseteq H^{*}\left(\operatorname{Alb} Z_{X}, \mathcal{H}_{X, j} \otimes P_{\eta}\right)
$$

(in fact, the first is a submodule of the second). By Step 3 and (**), it follows that

$$
\Phi_{\delta_{j}+\alpha} \widetilde{H}^{*}\left(\operatorname{Alb} Z_{X}, \mathcal{H}_{X, j} \otimes P_{\eta}\right)=\widetilde{H}^{*}\left(\operatorname{Alb} Z_{Y}, \mathcal{H}_{Y, j} \otimes P_{\bar{\varphi}(\eta)}\right) .
$$

By the projection formula on the decomposition (3.8), it follows that the sheaf $\mathcal{H}_{X, j} \otimes P_{\eta}$ is a direct summand of the sheaf $a_{f_{X *}}\left(a_{X *} \omega_{X} \otimes P_{\delta_{j}}^{\vee}\right) \otimes P_{\eta}$. Therefore, the module $H^{*}\left(\operatorname{Alb} Z_{X}, \mathcal{H}_{X, j} \otimes P_{\eta}\right)$ is a direct summand of the module $H^{*}\left(\operatorname{Alb} Z_{X}, a_{f_{X *}}\left(a_{X *} \omega_{X} \otimes P_{\delta_{j}}^{\vee}\right) \otimes P_{\eta}\right)$, which is 0-regular by Theorem 2.6, hence, in particular, generated in degree 0 . Hence, the module $H^{*}\left(\operatorname{Alb} Z_{X}\right.$, $\left.\mathcal{H}_{X, j} \otimes P_{\eta}\right)$ is generated in degree 0 as well, and we have equality in (3.11). By the same reasoning, the same thing happens for $Y$. Hence, Step 4 follows from (3.12).

Step 5: Conclusion of the proof. Let $q=\operatorname{dim} \operatorname{Alb} X=\operatorname{dim}$ Alb $Y$ (theorem of Popa-Schnell, [PS11]), and let $q^{\prime}=\operatorname{dim} \operatorname{Alb} Z_{X}=\operatorname{dim} \operatorname{Alb} Z_{Y}$ (Step 2). Note that since the quotient map $a_{f_{X}}$ has connected fibres, $R^{k} a_{f_{X} *} \mathcal{O}_{\mathrm{Alb} X}$ is a trivial bundle of rank $\left(\begin{array}{c}q-q^{\prime} \\ k\end{array}\right)$. Therefore, for $\eta \in \operatorname{Pic}^{0} Z_{X}$ such that $\widehat{a_{f_{X}}}(\eta)=\alpha+\delta_{j}$, we have

$$
\begin{aligned}
h^{i}\left(\operatorname{Alb} X, a_{X *} \omega_{X} \otimes P_{\alpha}\right) & \left.=\bigoplus_{\substack{k=0 \\
q-q^{\prime}}}^{i-k}\left(\operatorname{Alb} Z_{X}, R^{k} a_{f_{X *}}\left(a_{X *} \omega_{X}\right) \otimes P_{\eta}\right)\right) \\
& =\bigoplus_{k=0}^{q-q^{\prime}} h^{i-k}\left(\operatorname{Alb} Z_{X}, \mathcal{H}_{X, j} \otimes P_{\eta}\right)^{\oplus\left(\begin{array}{c}
q-q^{\prime} \\
k
\end{array}\right),}
\end{aligned}
$$

where the first equality is the Kollár decomposition (plus the projection formula) with respect to the morphism $a_{f_{X}}$ applied to the sheaf $a_{X *} \omega_{X}$ [Kol86b, Theorem 3.4] and the second equality follows from $(*)$ and the projection formula. The same formula holds for $Y$. Consequently, Theorem 1.1 follows from Step 4 applied to the last quantity.

\section{Application to irregular fibrations: Theorem 1.4}

Fibrations: Terminology. Let $X$ be a variety. A fibration of $X$ is an algebraic fibre space $g: X \rightarrow S$, where $S$ is a normal variety, called the base of the fibration. If a non-singular 


\section{F. CAucci and G. Pareschi}

model of $S$ (hence all of them) has maximal Albanese dimension, such a fibration is said to be irregular. A non-singular representative of a fibration of $X$ is a fibration $g^{\prime}: X^{\prime} \rightarrow S^{\prime}$ with both $X^{\prime}$ and $S^{\prime}$ smooth, equipped with birational morphisms $p: X^{\prime} \rightarrow X$ and $q: S^{\prime} \rightarrow S$ such that $g \circ p=q \circ g^{\prime}$. Two fibrations of $X$ are equivalent if there is a fibration $X^{\prime} \rightarrow S^{\prime}$ which is a birational representative for both of them.

Let $g$ be a fibration of $X$. We denote by $\operatorname{Pic}^{0}(g)$ the kernel of the restriction map from $\mathrm{Pic}^{0} X$ to the $\mathrm{Pic}^{0}$ of a general fibre. Notice that if $g^{\prime}$ is any non-singular representative of $g$, then $\operatorname{Pic}^{0}(g)=\operatorname{Pic}^{0}\left(g^{\prime}\right)$; therefore, $\operatorname{Pic}^{0}(g)$ depends only on the equivalence class of $g$. The group $\operatorname{Pic}^{0}(g)$ is an extension of $g^{*} \mathrm{Pic}^{0} S$ by a finite subgroup $\Gamma$ of $\mathrm{Pic}^{0} X / g^{*} \mathrm{Pic}^{0} S$ (see, for example, [Par17]); hence, it is disconnected unless $\Gamma=\hat{0}$.

Definition 4.1. Let $g: X \rightarrow S$ be an irregular fibration of $X$, and let us set $i=\operatorname{dim} X-\operatorname{dim} S$.

(a) The fibration $g$ is cohomologically non-detectable if $S$ birational to an abelian variety and $\operatorname{Pic}^{0}(g)$ is connected, and cohomologically detectable otherwise.

(b) The fibration $g$ is weakly- $\chi$-positive if there is a point $\alpha \in \mathrm{Pic}^{0} X$ such that for a non-singular representative $g^{\prime}: X^{\prime} \rightarrow S^{\prime}$ (hence for all of them, see Remark 4.2 below),

$$
\chi\left(R^{i} g_{*}^{\prime}\left(\omega_{X^{\prime}} \otimes P_{\alpha}\right)\right)>0 .
$$

Note that an $\alpha \in \operatorname{Pic}^{0} X$ as in the definition must belong to $\operatorname{Pic}^{0}(g)$. Therefore, one can always assume that $\alpha$ is a torsion point.

(c) The fibration $g$ is $\chi$-positive if for a non-singular representative $g^{\prime}$ as above (hence for all of them), $\chi\left(\omega_{S^{\prime}}\right)>0$.

Note that since $\omega_{S^{\prime}}=R^{i} g_{*}^{\prime} \omega_{X^{\prime}}$ (see [Kol86a, Proposition 7.6]), a $\chi$-positive irregular fibration is weakly- $\chi$-positive.

Remark 4.2. We keep the notation of Definition 4.1. From Hacon's generic vanishing (see Remark 2.4(1)) and an étale covering trick, it follows that $R^{i}\left(a_{S^{\prime}} \circ g^{\prime}\right)_{*}\left(\omega_{X^{\prime}} \otimes P_{\alpha}\right)$ is a GV-sheaf on Alb $S^{\prime}$. On the other hand, since $a_{S^{\prime}}$ is generically finite, by the combination of Kollár's vanishing and decomposition [Kol86b, Theorem 3.4], we have $R^{k} a_{S^{\prime} *} R^{h} g_{*}^{\prime}\left(\omega_{X^{\prime}} \otimes P_{\alpha}\right)=0$ for all $k>0$ and $h \geqslant 0$, hence $R^{i}\left(a_{S^{\prime}} \circ g^{\prime}\right)_{*}\left(\omega_{X^{\prime}} \otimes P_{\alpha}\right)=a_{S^{\prime} *} R^{i} g_{*}^{\prime}\left(\omega_{X^{\prime}} \otimes P_{\alpha}\right)$. Therefore, keeping in mind Remark 2.1, we see that condition (4.1) is equivalent to the condition

$$
V^{0}\left(S^{\prime}, R^{i} g_{*}^{\prime}\left(\omega_{X^{\prime}} \otimes P_{\alpha}\right)\right)=\operatorname{Pic}^{0} S^{\prime} .
$$

This in turn implies that condition (4.1) does not depend on the non-singular representative.

Preliminaries: The linearity theorem of Green and Lazarsfeld. The relation between the loci $V^{i}\left(X, \omega_{X}\right)$ and irregular fibrations follows from the fundamental theorem of Green and Lazarsfeld recalled below, with an addition of Simpson.

Theorem 4.3 ([GL91, Sim93]). Every irreducible component $W$ of the loci $V^{i}\left(X, \omega_{X}\right)$ is a linear subvariety, that is, a translate of an abelian subvariety $T \subset \operatorname{Pic}^{0} X$ by a torsion point. More precisely, let $\pi$ : Alb $X \rightarrow B:=\operatorname{Pic}^{0} T$ be the dual quotient. This defines the composed map $f: X \rightarrow B$

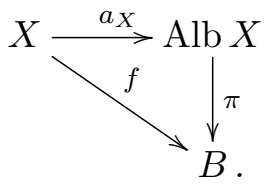




\section{DeRIVED INVARIANTS ARISING FROM the Albanese MAP}

Then there is a torsion ${ }^{5}$ element $\alpha \in \mathrm{Pic}^{0} X$ such that

$$
W=\widehat{\pi}\left(\operatorname{Pic}^{0} B\right)+\alpha .
$$

Moreover,

$$
\operatorname{dim} X-\operatorname{dim} f(X) \geqslant i
$$

Taking the Stein factorization of the map $f$, one gets a fibration $g: X \rightarrow S$, where $S$ is a normal projective variety of maximal Albanese dimension, and a finite morphism $a: S \rightarrow B$ such that $a \circ g=f$. Therefore, in our terminology, $g$ is an irregular fibration of $X$. We will refer to it as the fibration of $X$ induced by the component $W$ of $V^{i}\left(X, \omega_{X}\right)$, or also the fibration of $X$ induced by the abelian subvariety $T$ of $\mathrm{Pic}^{0} X$ parallel to the component $W$. In $[\mathrm{Par} 17$, Lemma 5.1], the following is shown, in particular.

Proposition 4.4. The above abelian variety $B$ is the Albanese variety of any non-singular model of $S^{\prime}$ of $S$, and the morphism a composed with the desingularization $S^{\prime} \rightarrow S$ is an Albanese morphism of $S^{\prime}$. In particular, $W=\widehat{\pi}\left(\mathrm{Pic}^{0} S^{\prime}\right)+\alpha$.

In conclusion, for a non-singular representative $g^{\prime}: X^{\prime} \rightarrow S^{\prime}$ of the induced fibration, we have the commutative diagram

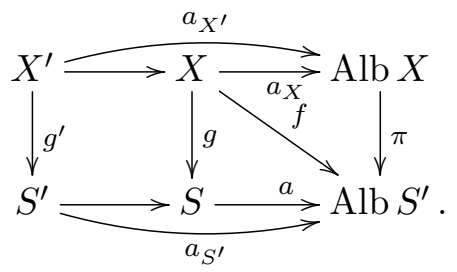

Preliminaries: Standard components and (weakly-) $\chi$-positive irregular fibrations. We will suppose henceforth that $X$ has maximal Albanese dimension. An irreducible component $W$ of $V^{i}\left(X, \omega_{X}\right)$ is said to be standard (see [Par17]) if there is equality in (4.4), that is,

$$
\operatorname{dim} X-\operatorname{dim} S=i \text {. }
$$

The relation between standard components and their induced fibrations is almost canonical. This is the content of the following lemma, inspired by [Lom18, Theorem 16]. In the statement, we consider the following sets:

- $\mathcal{A}(X)$ : the set of abelian subvarieties $T$ of the abelian variety $\operatorname{Pic}^{0} X$ such that some of their translates are standard components of $V^{i}\left(X, \omega_{X}\right)$ for some index $i$ (clearly, this can happen for only one index $i$, denoted by $i(T)$ ),

$-\mathcal{G}(X)$ : the set of equivalence classes of weakly- $\chi$-positive irregular fibrations of $\mathrm{X}$.

Lemma 4.5. The function $\sigma: \mathcal{A}(X) \rightarrow \mathcal{G}(X)$ taking an abelian subvariety to the class of its induced fibration (see the above paragraph) is a bijection. Moreover,

(a) the function $\sigma$ takes those abelian subvarieties which are themselves (standard) components of $V^{i}\left(X, \omega_{X}\right)$ to equivalence classes of $\chi$-positive fibrations;

(b) the base dimension of $\sigma(T)$ is $\leqslant \operatorname{dim} T$.

\footnotetext{
${ }^{5}$ This is due to Simpson.
} 


\section{F. CAucci and G. Pareschi}

Proof. First, we need to prove that if $T \in \mathcal{A}(X)$, then its induced fibration $g: X \rightarrow S$ is weakly$\chi$-positive. Let $i=i(T)$, and let $W$ be a component verifying (4.3), with $T=\operatorname{Pic}^{0} B=\operatorname{Pic}^{0} S^{\prime}$ (see Proposition 4.4 and (4.5). By the definition of the standard component, $\operatorname{dim} X-\operatorname{dim} S=i$. Thanks to the Kollár vanishing theorem [Kol86a, Theorem 2.1] and decomposition [Kol86b, Theorem 3.1], for a non-singular representative $g^{\prime}: X^{\prime} \rightarrow S^{\prime}$ of the fibration $g$, one has

$$
V^{i}\left(X^{\prime}, \omega_{X^{\prime}} \otimes P_{-\alpha}\right)=\bigcup_{j=0}^{i} \widehat{\pi}\left(V^{i-j}\left(S^{\prime}, R^{j} g_{*}^{\prime}\left(\omega_{X^{\prime}} \otimes P_{-\alpha}\right)\right)\right),
$$

where $\alpha \in \operatorname{Pic}^{0} X$ is the torsion point appearing in (4.3). Again by the Hacon generic vanishing theorem (Remark 2.4(1)) and an étale covering trick, codim $\operatorname{Pic}^{0} S^{\prime} V^{i-j}\left(S^{\prime}, R^{j} g_{*}^{\prime}\left(\omega_{X^{\prime}} \otimes P_{-\alpha}\right) \geqslant\right.$ $i-j$. Since, as we see using also Proposition 4.4, the left-hand side must contain $\widehat{\pi}\left(\operatorname{Pic}^{0} B\right)=$ $\widehat{\pi}\left(\operatorname{Pic}^{0} S^{\prime}\right)$, we have

that is, by Remark 4.2,

$$
V^{0}\left(S^{\prime}, R^{i} g_{*}^{\prime}\left(\omega_{X^{\prime}} \otimes P_{-\alpha}\right)\right)=\operatorname{Pic}^{0} S^{\prime}
$$

$$
\chi\left(R^{i} g_{*}^{\prime}\left(\omega_{X^{\prime}} \otimes P_{-\alpha}\right)\right)>0 .
$$

This proves the desired assertion. By the same steps in the reverse order, one proves that if $g: X \rightarrow S$ is a weakly- $\chi$-positive irregular fibration such that $\operatorname{dim} X-\operatorname{dim} S=i$, then (the equivalence class of $g$ induces standard components $W$ in $V^{i}\left(X, \omega_{X}\right)$ as follows. Assume that $-\alpha \in \operatorname{Pic}^{0}(g)$ is such that $\chi\left(R^{i} g_{*}^{\prime}\left(\omega_{X^{\prime}} \otimes a_{X}^{\prime} P_{-\alpha}\right)\right)>0$. Then

$$
\widehat{\pi}\left(\operatorname{Pic}^{0} S^{\prime}\right)+\alpha=\widehat{\pi}\left(V^{0}\left(S^{\prime}, R^{i} g^{\prime}\left(\omega_{X^{\prime}} \otimes P_{-\alpha}\right)\right)\right)+\alpha
$$

is a standard component of $V^{i}\left(X, \omega_{X}\right)$. It is clear that the two constructions above are inverse to each other. Properties (a) and (b) are clear.

Remark 4.6 (Cohomologically non-detectable fibrations). The above argument with the Kollár decomposition also proves that a cohomologically non-detectable irregular fibration $g: X \rightarrow S$ cannot be induced by a component $W$ of $V^{i}\left(X, \omega_{X}\right)$ of dimension at least $\operatorname{dim} X-i$. Indeed, for such a fibration, we have $V^{0}\left(S^{\prime}, \omega_{S^{\prime}}\right)=\{\hat{0}\}$ because $S$ is birational to an abelian variety. Therefore, since $\operatorname{Pic}^{0}(g)=g^{\prime *} \mathrm{Pic}^{0} S^{\prime}$, equality (4.7) cannot hold. Since we know that (4.7) holds as soon as $\operatorname{dim} X-\operatorname{dim} S=i$, it follows that $\operatorname{dim} X-\operatorname{dim} S<i$; that is, a component $W$ inducing such a fibration is non-standard. Moreover, since $\operatorname{dim} \operatorname{Alb} S^{\prime}=\operatorname{dim} S$, for such a component,

$$
\operatorname{dim} W<\operatorname{dim} X-i .
$$

This explains the terminology cohomologically non-detectable irregular fibration: either such a fibration is not induced by any component of $V^{i}\left(X, \omega_{X}\right)$ for some $i$ (as for example the projections of a product of elliptic curves), or such a component is non-standard.

At the opposite end, $\chi$-positive fibrations are the easiest to detect. The following proposition shows that equivalence classes of $\chi$-positive fibrations are derived invariants.

Proposition 4.7. Let $X$ and $Y$ be varieties of maximal Albanese dimension with equivalent derived categories. Then there is a base-preserving bijection between the sets of equivalence classes of $\chi$-positive irregular fibrations of $X$ and of $Y$.

Proof. By Lemma 4.5, all $\chi$-positive fibrations on a variety $X$ of maximal Albanese dimension are induced by abelian subvarieties which are (standard) components of $V^{i}\left(X, \omega_{X}\right)$ for some $i$. By Proposition 2.2(a), such components are contained in $V^{0}\left(X, \omega_{X}\right)$, hence in $\widehat{a_{f_{X}}}\left(\operatorname{Pic}^{0} Z_{X}\right)$ 


\section{DERIVED INVARIANTS ARISING FROM THE Albanese MAP}

(Theorem 3.3(b)). Therefore $\chi$-positive fibrations, as all fibrations induced by components of $V^{i}\left(X, \omega_{X}\right)$ for some $i$, factor, up to equivalence, through the Iitaka fibration $f_{X}: X \rightarrow Z_{X}$. But, by Orlov's theorem, a derived equivalence $\varphi: \mathbf{D}(X) \rightarrow \mathbf{D}(Y)$ induces an isomorphism of the canonical rings. Hence, the bases of the Iitaka fibrations $Z_{X}$ and $Z_{Y}$ are birational. As we are considering equivalence classes of fibration, we can assume $Z_{X}=Z_{Y}:=Z$. Therefore, the sets of equivalence classes of $\chi$-positive irregular fibrations of $X$ and $Y$ are both naturally bijective with the set of equivalence classes $\chi$-positive fibrations of $Z$.

Proof of Theorem 1.4. Let us recall that $b(X)$ denotes the minimal base dimension of the cohomologically detectable irregular fibrations of $X$.

Step 1. Assume $b(X)>0$.

(1) An irregular fibration $g$ of base dimension equal to $b(X)$ is cohomologically detectable if and only if it is weakly- $\chi$-positive. Moreover it is $\chi$-positive if and only if its base is not birational to an abelian variety.

(2) Conversely, every irreducible component $W$ of $V^{d-b(X)}\left(X, \omega_{X}\right)$ such that $\operatorname{dim} W \geqslant b(X)$ is standard. If this is the case, the abelian subvariety parallel to $W$ is also a component of $V^{d-b(X)}\left(X, \omega_{X}\right)$ if and only if the corresponding fibration (via Lemma 4.5) is $\chi$-positive.

The argument for Step 1 is well known to the experts (see, for example, [Par12, proof of Lemma 4.2]). We start with the following.

Claim 4.8. Let $g: X \rightarrow S$ be a cohomologically detectable irregular fibration such that $\operatorname{dim} X-$ $\operatorname{dim} S=i$. Then, keeping the notation above, for at least one $\alpha \in \operatorname{Pic}^{0}(g)$, the locus

$$
V^{0}\left(S^{\prime}, R^{i} g_{*}^{\prime}\left(\omega_{X^{\prime}} \otimes P_{\alpha}\right)\right)
$$

is positive-dimensional.

Proof. We first observe that if $\alpha$ belongs to a component of $\operatorname{Pic}^{0}(g)$ different from the neutral one, then the locus (4.9) is positive-dimensional. In fact, it must be non-empty thanks to Proposition 2.2(b), and if it was 0-dimensional, this would induce via Remark 2.4(3) a (0-dimensional) component of the locus $V^{q\left(S^{\prime}\right)}\left(R^{i} g_{*}^{\prime}\left(\omega_{X^{\prime}} \otimes P_{\alpha}\right)\right.$. This would imply that $\operatorname{dim} S^{\prime}=q\left(S^{\prime}\right)$ and, via the ever-present Kollár decomposition as in (4.6), this would induce some elements different from $\{\hat{0}\}$ in the locus $V^{d}\left(X, \omega_{X}\right)$, which is impossible.

Therefore we are left with the case when $\operatorname{Pic}^{0}(g)$ is connected and $V^{0}\left(S^{\prime}, \omega_{S}^{\prime}\right)$ is 0-dimensional (recall that $R^{i} g_{*}^{\prime} \omega_{X^{\prime}}=\omega_{S^{\prime}}$ ). But this, by a theorem of Ein-Lazarsfeld [CH01a, Theorem 1.8], is equivalent to the fact that $S^{\prime}$ is birational to an abelian variety; that is, the fibration would be non-detectable.

We now turn to Step 1(1). Let $g: X \rightarrow S$ be a cohomologically detectable fibration with $\operatorname{dim} S=b(X)$. We claim that if it is not weakly- $\chi$-positive, then there is another cohomologically detectable fibration of lower base dimension factoring (up to equivalence) through $g$, in contradiction with the definition of $b(X)$. Let $\alpha \in \mathrm{Pic}^{0}(g)$ be as in Claim 4.8. Then, again by Remark 2.4(3), the irreducible components of (4.9) of codimension $c$, with $0<c<q\left(S^{\prime}\right)$, are also irreducible components of $V^{c}\left(S^{\prime}, R^{i} g_{*}^{\prime}\left(\omega_{X^{\prime}} \otimes P_{\alpha}\right)\right)$, where $i=d-\operatorname{dim} S$. Via the Kollár decomposition, they induce positive-dimensional components of the locus $V^{i+c}\left(\omega_{X}\right)$. Via the linearity theorem and Remark 4.6, such a component induces another cohomologically detectable irregular fibration of $X$, say $h$, with $d-\operatorname{dim} h(X) \geqslant i+c=d-\operatorname{dim} S+c$. Hence $\operatorname{dim} h(X) \leqslant \operatorname{dim} S-c$, as 


\section{F. Caucci and G. Pareschi}

asserted. This proves the direct implication of the first equivalence of (1). The other implication is clear. Passing to the second equivalence, the direct implication is clear. Conversely, let us suppose that the base is non-birational to an abelian variety. Then, by the theorem of Ein-Lazarsfeld as above, $V^{0}\left(S^{\prime}, \omega_{S^{\prime}}\right)$ is positive-dimensional. If it was strictly contained in $\mathrm{Pic}^{0} S^{\prime}$, then, as above, its components would induce a cohomologically detectable fibration $h$ of smaller base dimension, against the definition of $b(X)$. This completes the proof of part (1).

Passing to Step $1(2)$, let $W$ be a positive-dimensional component of $V^{d-b(X)}\left(X, \omega_{X}\right)$ such that $\operatorname{dim} W \geqslant b(X)$. The statement to prove is that the induced fibration $g$ has base dimension equal to $b(X)$. If the base dimension was less than $b(X)$, then, by the definition of the integer $b(X)$, the fibration $g$ would be cohomologically non-detectable. This means that the base would be birational to an abelian variety of dimension less than $b(X)$, and therefore, by (4.3), the component $W$ would have dimension less than $b(X)$. The last assertion follows from the second equivalence of part (1) via Lemma 4.5. This concludes the proof of Step 1.

Step 2: Conclusion of the proof of Theorem 1.4.

Claim 4.9. We have $b(X)>0$ if and only if $\operatorname{dim} V^{i}\left(X, \omega_{X}\right) \geqslant d-i$ for some $0<i<d$. If $b(X)>0$, then $d-b(X)$ is the maximal index $i$ with $0<i<d$ such that $\operatorname{dim} V^{i}\left(X, \omega_{X}\right) \geqslant d-i$.

Proof. Concerning the first equivalence, if $b(X)>0$, then by Step 1(1), there is a weakly- $\chi$ positive fibration $g$ of base dimension $b(X)$, and therefore, by Lemma 4.5, there is a component of $V^{d-b(X)}\left(X, \omega_{X}\right)$ of dimension at least $b(X)$. The other implication follows from Remark 4.6. The last assertion follows by the same reasoning.

Now, let $X$ and $Y$ be derived-equivalent varieties. By Claim 4.9, the integers $b(X)$ and $\mathrm{b}(\mathrm{Y})$ are respectively determined by the dimensions of the various loci $V^{i}\left(X, \omega_{X}\right)$ and $V^{i}\left(Y, \omega_{Y}\right)$. Therefore, Corollary 1.3 yields that $b(X)=b(Y):=b$. From Step 1, cohomologically detectable fibrations of base dimension equal to $b$ are weakly- $\chi$-positive, and their equivalence classes correspond to all components of $V^{d-b}\left(X, \omega_{X}\right)$ of dimension at least $b$, and such components are standard. Therefore, by Lemma 4.5, they are in a 1-1 correspondence with the corresponding subset of abelian subvarieties of $\operatorname{Pic}^{0} X$. The same holds for $Y$. Therefore, by Corollary 1.3, the Rouquier isomorphism induces a bijection between the sets of equivalence classes of cohomologically detectable fibrations of base dimension $b$ on $X$ and on $Y$.

It remains to prove that there is a bijection preserving, up to equivalence, the bases of the fibrations. ${ }^{6}$ To begin, we note that the above-constructed bijection is base preserving on the subset of fibrations whose bases are birational to abelian varieties. Indeed, Step 1 shows that they correspond to components of $V^{d-b}\left(X, \omega_{X}\right)$ of dimension at least $b$ such that their parallel abelian varieties, namely $g^{\prime *} \operatorname{Pic}^{0} S^{\prime}$, are not components of $V^{d-b}\left(X, \omega_{X}\right)$. The same holds for $Y$. The Rouquier isomorphism sends isomorphically such components of $V^{d-b}\left(X, \omega_{X}\right)$ to components of $V^{d-b}\left(Y, \omega_{Y}\right)$, say $h^{\prime *} \mathrm{Pic}^{0} R^{\prime}$, with the same property. Both $S^{\prime}$ and $R^{\prime}$ are birational to abelian varieties, and their Picard tori are isomorphic. Therefore, $S^{\prime}$ is birational to $R^{\prime}$. Concerning the remaining fibrations, namely those whose bases are not birational to abelian varieties, by Step 1(1) they are $\chi$-positive. Therefore, Proposition 4.7 applies. (Here we are not claiming that this bijection coincides with the one constructed above, namely the one induced by the Rouquier

\footnotetext{
${ }^{6}$ Our notion of equivalence of fibrations is weaker than Lombardi's notion of isomorphism of irrational pencils [Lom18]. However, as in Lombardi's paper, it can be proved that the bijection of Theorem 1.4 is base preserving not only up to equivalence but also up to isomorphism of the bases of the Stein factorizations of the maps $f$ of (4.5).
} 


\section{DERIVED INVARIANTS ARISING From the Albanese MAP}

isomorphism. However, this is true, but the proof of this fact requires some tools beyond those used in this paper.)

\section{ACKNOWLEDGEMENTS}

We thank Luigi Lombardi for answering our questions and the referee for his careful reading and his helpful comments to improve the exposition of this text.

\section{REFERENCES}

Abu17 R. Abuaf, Homological units, Int. Math. Res. Not. 2017 (2017), no. 22, 6943-6960; doi:10. 1093/imrn/rnw170.

Bea92 A. Beauville, Annulation du $H^{1}$ pour les fibrés en droites plats, Complex Algebraic Varieties (Bayreuth, 1990), Lecture Notes in Math., vol. 1507 (Springer, Berlin, 1992), 1-15; doi:10. $1007 / \mathrm{BFb} 0094507$.

Căl03 A. Căldăraru, The Mukai pairing, I: The Hochschild structure, 2003, arXiv:math.AG/0308079.

Cas05 G. Castelnuovo, Sulle superficie aventi il genere aritmetico negativo, Rend. Circ. Mat. Palermo 20 (1905), no. 1, 55-60; doi:10.1007/BF03014028.

CH01a J.A. Chen and C. Hacon, Characterization of abelian varieties, Invent. Math. 143 (2001), no. 2, 435-447; doi:10.1007/s002220000111.

CH01b _ Pluricanonical maps of varieties of maximal Albanese dimension, Math. Ann. 320 (2001), no. 2, 367-380; doi:10.1007/PL00004478.

CJ18 J.A. Chen and Z. Jiang, Positivity in varieties of maximal Albanese dimension, J. reine angew. Math. 736 (2018), 225-253; doi:10.1515/crelle-2015-0027.

EL97 L. Ein and R. Lazarsfeld, Singularities of theta divisors and the birational geometry of irregular varieties, J. Amer. Math. Soc. 10 (1997), no. 1, 243-258; doi:10.1090/S0894-0347-97-00223-3.

dFr05 M. de Franchis, Sulle superficie algebriche le quali contengono un fascio irrazionale di curve, Rend. Circ. Mat. Palermo 20 (1905), no. 1, 49-54; doi:10.1007/BF03014027.

GL91 M. Green and R. Lazarsfeld, Higher obstructions to deforming cohomology groups of line bundles, J. Amer. Math. Soc. 4 (1991), no. 1, 87-103; doi:10.2307/2939255.

Hac04 C. Hacon, A derived category approach to generic vanishing, J. reine angew. Math. 575 (2004), 173-187; doi:10.1515/crll.2004.078.

HPS18 C. Hacon, M. Popa, and C. Schnell, Algebraic fiber spaces over abelian varieties: around a recent theorem by Cao and Păun, in Local and Global Methods in Algebraic Geometry, Contemp. Math., vol. 712 (Amer. Math. Soc., Providence, RI, 2018), 143-195; doi:10.1090/conm/712/14346.

Huy06 D. Huybrechts, Fourier-Mukai transforms in algebraic geometry, Oxford Math. Monogr. (The Clarendon Press, Oxford Univ. Press, Oxford, 2006); doi:10.1093/acprof:oso/ 9780199296866.001 .0001$.

Kol86a J. Kollár, Higher direct images of dualizing sheaves. I, Ann. of Math. 123 (1986), no. 1, 11-42; doi:10.2307/1971351.

Kol86b_ Higher direct images of dualizing sheaves. II, Ann. of Math. 124 (1986), no. 1, 171-202; doi:10.2307/1971390.

Lom14 L. Lombardi, Derived invariants of irregular varieties and Hochschild homology, Algebra Number Theory 8 (2014), no. 3, 513-542; doi:10.2140/ant.2014.8.513.

Lom18_, Derived equivalence and fibrations over curves and surfaces, 2018, arXiv:1803.08656.

LP10 R. Lazarsfeld and M. Popa, Derivative complex, BGG correspondence, and numerical inequalities for compact Kähler manifolds, Invent. Math. 182 (2010), no. 3, 605-633; doi:10.1007/ s00222-010-0269-4. 


\section{F. CAUCCI AND G. PAREschi}

LP15 L. Lombardi and M. Popa, Derived equivalence and non-vanishing loci II, Recent Advances in Algebraic Geometry, London Math. Soc. Lecture Note Ser., vol. 417 (Cambridge Univ. Press, Cambridge, 2015), 291-306; doi:10.1017/CB09781107416000.016.

LPS11 R. Lazarsfeld, M. Popa, and C. Schnell, Canonical cohomology as an exterior module, Pure Appl. Math. Q. 7 (2011), no. 4, 1529-1542; doi:10.4310/PAMQ.2011.v7.n4.a21.

Orl03 D. O. Orlov, Derived categories of coherent sheaves and equivalences between them, Russian Math. Surveys 58 (2003), no. 3, 511-591; doi:10.1070/RM2003v058n03ABEH000629.

Par12 G. Pareschi, Basic results on irregular varieties via Fourier-Mukai methods, in Current Developments in Algebraic Geometry, Math. Sci. Res. Inst. Publ., vol. 59 (Cambridge Univ. Press, Cambridge, 2012), 379-403.

Par17 Standard canonical support loci, Rend. Circ. Mat. Palermo 66 (2017), no. 1, 137-157; doi:10.1007/s12215-016-0269-3.

Pop13 M. Popa, Derived equivalence and non-vanishing loci, in A Celebration of Algebraic Geometry, Clay Math. Proc., vol. 18 (Amer. Math. Soc., Providence, RI, 2013), 567-575.

PPS17 G. Pareschi, M. Popa, and C. Schnell, Hodge modules on complex tori and generic vanishing for compact Kähler manifolds, Geom. Topol. 21 (2017), no. 4, 2419-2460; doi:10.2140/gt. 2017. 21.2419.

PS11 M. Popa and C. Schnell, Derived invariance of the number of holomorphic 1-forms and vector fields, Ann. Sci. Éc. Norm. Supér. (4) 44 (2011), no. 3, 527-536; doi:10.24033/asens. 2149.

PS13 G Generic vanishing theory via mixed Hodge modules, Forum Math. Sigma 1 (2013), e1; doi:10.1017/fms.2013.1.

Rou11 R. Rouquier, Automorphismes, graduations et catégories triangulées, J. Inst. Math. Jussieu 10 (2011), no. 3, 713-751; doi:10.1017/S1474748011000089.

Sim93 C. Simpson, Subspaces of moduli spaces of rank one local systems, Ann. Sci. École Norm. Sup. (4) 26 (1993), no. 3, 361-401; doi:10.24033/asens. 1675.

Swa96 R. G. Swan, Hochschild cohomology of quasiprojective schemes, J. Pure Appl. Algebra 110 (1996), no. 1, 57-80; doi:10.1016/0022-4049(95)00091-7.

Federico Caucci caucci@mat.uniroma1.it

Sapienza Università di Roma, P.le Aldo Moro 5, I-00185 Roma, Italy

Giuseppe Pareschi pareschi@mat.uniroma2.it

Università di Roma Tor Vergata, V.le della Ricerca Scientifica, I-00133 Roma, Italy 